\title{
INVARIANCE OF THE ADMISSIBILITY OF NUMBERS UNDER CERTAIN GENERAL TYPES OF TRANSFORMATIONS*
}

\author{
BY \\ T. N. E. GREVILLE
}

Most physical events can be resolved, in theory at least, into a set of independent components in such a way that, when the result of each component event is known, the result of the principal event is fully determined. If the question of order does not enter into the determination so that, without changing the situation, the component events may be thought of as occurring simultaneously, the relation between the component events and the principal event can be formulated analytically in the "Verbindung" operation of von Mises. However, if the order of the component events is significant-as, for example, when the principal event is a set of tennis, the outcome depending to some extent on the order of gains and losses in a series of individual games-von Mises points out that his methods are not applicable. $\dagger$ It is the purpose of this paper to develop a set of transformations capable of dealing with such problems.

There will be associated with each transformation of this set a set of admissible numbers having the power of the continuum for which the property of admissibility is invariant under the given transformation. Furthermore, every denumerable subset of such transformations will be shown to have a similar property. The meaning of admissibility may be explained as follows. Assume that a one-to-one correspondence has been established between the set of all positive integers $\lambda$ and the set of all sets of integers $n, r_{1}, r_{2}, \cdots, r_{k}$, such that $0<r_{1}<r_{2}<\cdots<r_{k} \leqq n$. Let the digits of a number $u$ (having as digits only zeros and ones) be divided into consecutive, nonoverlapping groups of $n$ digits each. Let $T_{\lambda}$ denote the transformation which transforms $u$ into a number $v$ constituted as follows: $v$ contains a single digit corresponding to each group of $n$ digits of $u$. This digit is a one only if the $r_{1}$ th, $r_{2}$ th, $\cdots, r_{k}$ th digits in the corresponding group of digits of $u$ are all ones; otherwise, it is a zero. If $p(x)$ denotes the limit of the relative frequency of ones in the number

* Presented to the Society, June 22, 1933; received by the editors March 30,1939. This paper is a dissertation submitted in partial fulfillment of the requirements for the degree of doctor of philosophy in the University of Michigan. The writer wishes to express his appreciation to Professor A. H. Copeland for his valuable assistance in the preparation of this paper.

$\dagger$ von Mises [2, pp. 108-109]. References to literature are given at the end of this paper. 
$x$, then $u$ is said to be admissible if $p\left[T_{\lambda}(u)\right]$ is equal to $p^{k}(x)$ for every $\lambda .^{*}$

The set of transformations to be considered herein includes as special cases all the transformations $T_{\lambda}$ - that is, the set of operations employed by Copeland as the fundamental set for admissible numbers. By a proper choice of the fundamental set, a class of numbers having more general properties than the admissible number can be defined, and it will be shown that numbers belonging to such classes actually exist. These transformations represent certain processes followed in the classical methods of computing probabilities, and, since their properties are arrived at through rigorous mathematical developments without any assumption of "equal likelihood," they furnish a new kind of justification for the use of the customary methods in calculating the probabilities of events consisting of combinations of other events. As a byproduct of more general theorems, the existence of admissible numbers having all possible probabilities is demonstrated by a new method. This also constitutes a proof of the existence of the "normale Folge" of Reichenbach, as the latter has been proved equivalent to the admissible number.

When the theory of probability is resorted to in a practical situation, it is not, as a rule, because the events under consideration are believed to be governed altogether by chance, but because there are no data, except of a statistical nature, on which to base a prediction. However, it is conceivable, in the light of modern physical researches in the quantum theory and along other lines, that the result of analyzing an event into its ultimate constituents might be a set of independent events, each governed entirely by chance. Should this prove to be the case, the admissibility of physical events would depend definitely on the invariance of admissibility under the "Verbindung" operation and under the transformations here considered, since chance events could be expected to satisfy the conditions of admissibility. The study of the relation between a given event and the set of component events of which it is made up is facilitated by certain group properties possessed by various sets of transformations which will be dealt with. The property by which the resultant of any pair (and therefore any finite number) of transformations of a given set is itself a transformation of the set will be designated as the property $G$. This property is possessed by the four fundamental operations of von Mises, and by the set which is the subject of this paper, as well as by a number of special subsets, including the fundamental set for admissible numbers. It is evident that the product of any finite number of sets of transformations having the property $G$ has the property $G$.

* $T_{\lambda}(u)=\left[u \subset\left(r_{1}-1, n\right)\right] \cdot\left[u \subset\left(r_{2}-1, n\right)\right] \cdots\left[u \subset\left(r_{k}-1, n\right)\right]$. In connection with the notation, see Copeland [1, 6]. 
1. The general transformation of the set $R$. Let certain special permutations of zeros and ones in the digits of a number $u$ be associated with digits 1 of a number $y$, and let other such permutations be associated with digits 0 of $v$. Such a set of permutations may be used to define a transformation on the number $u$, giving rise to the number $v$. Only permutations of finite length will be considered. Let the digits of $u$ be divided into a sequence of mutually exclusive groups of successive digits in the following manner. If no finite number of successive digits of $u$, starting with the first digit, constitutes one of the special designated permutations of zeros and ones, the entire number $u$ is considered as a single group. Otherwise, the first group consists of the smallest number of successive digits, starting with the first, which together constitute one of the specified permutations. The second group, if any, is defined in the same way, except that it begins with the digit immediately following the last digit of the preceding group, rather than with the first digit of $u$. Subsequent groups are similarly defined. It follows that every digit of $u$ belongs to one and only one group, and that either the number of groups is infinite, or else there is a last group containing an infinite number of digits (provided, of course, the number $u$ itself contains an infinite number of digits). To each group (except the last, if any) there corresponds a digit of $v$ whose value ( 0 or 1$)$ is that associated with the permutation formed by the group. If there is a last group, the number of digits of $v$ is finite; otherwise, it is infinite. The number $v$ obtained by this process is unique, although the converse is not true.

An illustration of a problem for which the transformations given by other writers are not adequate is the rubber of bridge, which is won by the side winning two out of three games. In this case, the " 1 permutations" are 11, 101 , and 011 , while the " 0 permutations" are 00,010 , and 100 . If

$$
u=1010011101010 \cdots,
$$

we should divide $u$ into groups as follows:

$$
u=101 / 00 / 11 / 101 / 010 / \cdots,
$$

and we should have $v=10110 \cdots$.

Wald* also makes use of the notion of " 0 permutations" and " 1 permutations" in determining a new number whose digits depend on those of an original "collective." My procedure differs from his in two respects which are essential to the developments in this paper. First, Wald's permutations overlap, each commencing with the first term of the collective, while mine are nonoverlapping, each commencing from the end of the preceding one. Sec-

* Wald [1]. 
ondly, Wald uses the derived number solely for the purpose of making a selection (Auswahl) from the original collective, and considers only the properties of the selected sequence $u \subset v$, while I am concerned with the properties of the number $v$ itself.

My transformation is also similar to the type employed by Reichenbach* as the fundamental set for the "normale Folge." Reichenbach's transformation is a selection which selects every digit preceded by any one of certain specified permutations. In my method, this type of selection can be approximated by a transformation defined by means of permutations consisting of the permutations associated with Reichenbach's transformation followed by a single zero or one. My transformations are more general in that they are not restricted to mere selections; his are more general in that the permutations formed from the digits of the number may overlap, and need not be consecutive.

It is obvious from the nature of the transformation that any specified permutation is superfluous which contains another specified permutation as a group of successive digits, beginning with the first. For simplicity, it will be assumed that such redundant permutations are not used. A transformation $T$, defined by means of two sets of specified permutations in the manner indicated, is said to belong to the set $R$ if the set consisting of all the specified permutations (the sum of the " 0 " and " 1 " sets) satisfies the following condition, which will be called the condition of indeterminacy.

Any permutation whatever of zeros and ones of finite length either (i) is itself a specified permutation, or (ii) contains a specified permutation as a group of successive digits, beginning with the first, or (iii) is contained in a specified permutation as a group of successive digits, beginning with the first.

The meaning of this condition is that, no matter how many digits of $u$ in any group have been considered without obtaining a specified permutation, there is always a possibility that by considering more digits a specified permutation will be obtained.

The set $P^{(T)}$ of specified permutations associated with the transformation $T$ is made up of the two sets $P_{1}{ }^{(T)}$ and $P_{0}{ }^{(T)}$, which consist of permutations associated with the digits 1 and 0 , respectively, in the number $v=T(u)$. It will be assumed that, in all the transformations discussed, neither $P_{1}^{(T)}$ nor $P_{0}^{(T)}$ is vacuous.

Associated with every transformation $T$ of $R$ are the probability functions

$$
\pi^{(T)}(p)=\sum_{(h, k)} \xi_{h k}^{(T)} p^{h} q^{k}, \quad \rho^{(T)}(p)=\sum_{(h, k)} \omega_{h k}^{(T)} p^{h} q^{k},
$$

* Reichenbach [1]. 
in which $\xi_{h k}^{(T)}$ denotes the number of permutations of $P_{1}^{(T)}$ which consist of exactly $h 1$ 's and $k 0$ 's, $\omega_{h k}^{(T)}$ denotes the number of such permutations in $P_{0}{ }^{(T)}$, and $\sum_{(h, k)}$ denotes summation over all nonnegative integral values of $h$ and $k$. It should be noted that $\pi^{(T)}(p)$ and $\rho^{(T)}(p)$ are exactly the expressions which would be obtained by a priori methods for the probabilities of occurrence of the digits 1 and 0 , respectively, in the number $v=T(u)$.

A transformation $T$ of $R$ is said to be $a d m i s s i b l e$, or to belong to the set $R_{a}$, if

$$
\pi^{(T)}(p)+\rho^{(T)}(p) \equiv 1
$$

identically in $p$. It is problematical whether it is a sufficient condition for admissibility to have this equality hold for a set of values of $p$ everywhere dense on the unit interval.

A transformation $T$ of $R$ is said to be finite (or to belong to the set $R_{f}$ ) if $P^{(T)}$ is finite.

A transformation $T$ of $R$ is said to be symmetric (or to belong to the set $R_{s}$ ) if the identity

$$
\pi^{(T)}(p) \equiv{ }_{p} \quad \rho^{(T)}(q)
$$

is satisfied. The physical interpretation of a symmetric transformation in connection with a series of games is that a player's probability of winning a rubber or set of games is related in the same way to the probability of his winning a single game, regardless of which side he takes. A sufficient but not a necessary condition that a transformation $T$ be symmetric is that $\xi_{h k}^{(T)}=\omega_{k h}^{(T)}$ for every pair of values of $h$ and $k$. The physical meaning of the latter condition would be that the rules of the rubber or set are precisely the same for both players. If $T$ belongs to $R_{s}$,

$$
\pi^{(T)}(1 / 2)=\rho^{(T)}(1 / 2) .
$$

It is evident that the sets $R, R_{f}$, and $R_{s}$ have the property $G$. The set $R_{a}$ will now be shown also to possess this property.

THEOREM 1. If $T$ and $T^{\prime}$ are any two transformations of $R_{a}$, and $T^{\prime \prime}$ is a transformation such that $T^{\prime \prime}(u)=T^{\prime}[T(u)]$, then $T^{\prime \prime}$ belongs to $R_{a}$.

We note that

$$
\begin{aligned}
\pi^{\left(T^{\prime}\right)}\left[\pi^{(T)}(p)\right] & =\sum_{(h, k)} \xi_{h k}^{\left(T^{\prime}\right)}\left[\sum_{(\gamma, \delta)} \xi_{\gamma \delta}^{(T)} p^{\gamma} q^{\delta}\right]^{h}\left[\sum_{(\gamma, \delta)} \omega_{\gamma \delta}^{(T)} p^{\gamma} q^{\delta}\right]^{k} \\
& =\sum_{(h, k)} \xi_{h k}^{\left(T^{\prime}\right)}\left\{\sum^{\gamma \delta} \prod_{i=1}^{h} \xi_{\gamma_{i} \delta_{i}}^{(T)} p^{\gamma_{i} \delta_{i}} q^{k} \prod_{j=1}^{k} \omega_{\gamma_{j^{\prime}} \delta^{\prime}{ }^{\prime}}^{(T)} p^{\left.\gamma_{j^{\prime}}{ }^{\delta_{j^{\prime}}}\right\}} q^{(}\right.
\end{aligned}
$$

where $\sum^{\gamma^{\delta}}$ denotes summation over all possible choices of the $\gamma_{i}, \delta_{i}, \gamma_{i}{ }^{\prime}$, and 
$\delta_{i}{ }^{\prime}$. The latter expression may be rewritten in the form

$$
\sum^{\gamma \delta h k} \xi_{h k}^{\left(T^{\prime}\right)}\left[\prod_{i=1}^{h} \xi_{\gamma_{i} \delta_{i}}^{(T)} \prod_{j=1}^{k} \omega_{\gamma_{j^{\prime}} \delta_{j^{\prime}}}^{(T)}\right] p^{m} q^{n}
$$

where $m=\sum_{i=1}^{h} \gamma_{i}+\sum_{j=1}^{k} \gamma_{j}{ }^{\prime}$ and $n=\sum_{i=1}^{h} \delta_{i}+\sum_{j=1}^{k} \delta_{j}{ }^{\prime}$. Consider, for a moment, only those terms in the summation such that $m=\mu$ and $n=\nu$. If the summation is restricted to these terms,

$$
\sum^{\gamma \delta h k} \xi_{h k}^{\left(T^{\prime}\right)}\left[\prod_{i=1}^{h} \xi_{\gamma_{i} \delta_{i}}^{(T)} \prod_{j=1}^{k} \omega_{\gamma_{j^{\prime}} \delta^{\prime}}^{(T)}\right]
$$

is the total number of arrangements of $\mu$ 1's and $\nu 0$ 's of $u$ which will give rise to a digit 1 of $T^{\prime \prime}(u)$. Therefore, the above expression can be written

$$
\sum_{(\mu, \nu)} \xi_{\mu \nu}^{\left(T^{\prime \prime}\right)} p^{\mu} q^{\nu}=\pi^{\left(T^{\prime \prime}\right)}(p) .
$$

Similarly, it can be shown that

Hence,

$$
\rho^{\left(T^{\prime}\right)}\left[\pi^{(T)}(p)\right]=\rho^{\left(T^{\prime \prime}\right)}(p) .
$$

$$
\pi^{\left(T^{\prime \prime}\right)}(p)+\rho^{\left(T^{\prime \prime}\right)}(p)=\pi^{\left(T^{\prime \prime}\right)}\left[\pi^{(T)}(p)\right]+\rho^{\left(T^{\prime}\right)}\left[\pi^{(T)}(p)\right] \equiv_{p} 1,
$$

since $T^{\prime}$ belongs to $R_{a}$. This proves the theorem.

2. Relation between probability and measure. The probability functions $\pi^{(T)}(p)$ and $\rho^{(T)}(p)$ are closely related to the measure of certain sets of numbers. Consider the case in which $p$ has a rational value $\beta / \alpha$, and let a number $y$ in the scale of notation with radix $\alpha$ be so related to the binary number $u$ that $u^{(i)}=1$ if $y^{(i)}=0,1,2, \cdots, \beta-1$, and $u^{(i)}=0$ otherwise. The number $\beta$ is not allowed to have the values 0 and $\alpha$, the rational probabilities zero and unity being excluded from consideration.

Theorem 2. If $T$ is any transformation of $R$, and a set $E$ consists of the numbers $y$ in the scale of $\alpha(p=\beta / \alpha)$ associated with the set of all numbers $u$ such that the first $\mu+\nu$ digits of $v=T(u)$ exist and consist of $\mu$ 1's and $\nu 0$ 's in a prescribed order, then

$$
m(E)=\left[\pi^{(T)}(p)\right]^{\mu}\left[\rho^{(T)}(p)\right]^{\nu} .
$$

Let us fix our attention on a particular $u$ such that the first $\mu+\nu$ digits of the corresponding $v$ are in the prescribed order. Let us suppose that $\gamma_{h k}$ of the first $\mu$ 1's of the digits of $v$ result from those permutations of the digits of $u$ which consist of $h 1$ 's and $k 0$ 's, and that $\delta_{h k}$ of the first $\nu 0$ 's of $v$ result from such permutations. Then $\sum_{(h, k)} \gamma_{h k}=\mu$ and $\sum_{(h, k)} \delta_{h k}=\nu$, and 
$\sum_{(h, k)}\left(\gamma_{h k}+\delta_{h k}\right)(h+k)$ digits of $u$ (and hence of $y$ ) are required to produce these prescribed $\mu+\nu$ digits of $v$.

Next, let us form arbitrary decompositions of $\mu+\nu$ into sums of nonnegative integers $\gamma_{h k}$ and $\delta_{h k}$, respectively. We can assign the number pair $(h, k)$ to any $\gamma_{h k}$ of the $\mu$ 1's of the digits of $v$. A digit to which $(h, k)$ is assigned is required to be produced by $h 1$ 's and $k 0$ 's of the digits of $u$. The total number of possible assignments is

$$
\frac{\mu !}{\prod_{(h, k)} \gamma_{h k} !} .
$$

The number of such assignments with respect to the $\nu 0$ 's of $v$ is

$$
\frac{\nu !}{\prod_{(h, k)} \delta_{h k} !}
$$

Hence, under the above decomposition, the number of ways in which the first $\sum_{(h, k)}\left(\gamma_{h k}+\delta_{h k}\right)(h+k)$ digits of $y$ can be chosen so as to produce the prescribed $\mu+\nu$ digits of $v$ is

$$
\frac{\mu !}{\prod_{(h, k)} \gamma_{h k} !} \frac{\nu !}{\prod_{(h, k)} \delta_{h k} !} \prod_{(h, k)}\left[\xi_{h k}^{(T)} \beta^{h}(\alpha-\beta)^{k}\right]^{\gamma_{h k}} \prod_{(h, k)}\left[\omega_{h k}^{(T)} \beta^{h}(\alpha-\beta)^{k}\right]^{\delta_{h k}}
$$

It will be observed that if one of the integers $\xi_{h k}(T)$ is zero and the corresponding integer $\gamma_{h k}$ is not zero, then there are no ways in which the digits of $y$ can be chosen so as to produce the prescribed $\mu+\nu$ digits of $v$. If, however, both $\xi_{h k}{ }^{(T)}$ and the corresponding $\gamma_{h k}$ are zero, then the ambiguous symbol $0^{\circ}$ must be assigned the value 1 in order for the formula to be correct. The same is true of $\omega_{h k}^{(T)}$ and $\delta_{h k}$.

Since the measure of the set of points corresponding to the set of numbers $y$ for which the first $\sum_{(h, k)}\left(\gamma_{h k}+\delta_{h k}\right)(h+k)$ digits are prescribed is $\alpha^{-c}$, where $c=\sum_{(h, k)}\left(\gamma_{h k}+\delta_{h k}\right)(h+k)$, it follows that the measure of $E$ is

$$
\begin{aligned}
\sum \sum\left\{\frac{\mu !}{\prod_{(h, k)} \gamma_{h k} !}\right. & \left.\frac{\nu !}{\prod_{(h, k)} \delta_{h k} !} \prod_{(h, k)}\left[\xi_{h k}^{(T)} \beta^{h}(\alpha-\beta)^{k}\right]^{\gamma_{h k}} \prod_{(h, k)}\left[\omega_{h k}^{(T)} \beta^{h}(\alpha-\beta)^{k}\right]^{\delta_{h k}} \alpha^{-c}\right\} \\
& =\sum \sum\left\{\frac{\mu !}{\prod_{(h, k)} \gamma_{h k} !} \frac{\nu !}{\prod_{(h, k)} \delta_{h k} !} \prod_{(h, k)}\left[\xi_{h k}^{(T)} p^{h} q^{k}\right]^{\gamma_{h k}} \prod_{(h, k)}\left[\omega_{h k}^{(T)} p^{h} q^{k}\right]^{\delta_{h k}}\right\},
\end{aligned}
$$

where the expressions in braces are to be summed for all possible decomposi- 
tions of $\mu$ and $\nu$. After application of the multinomial theorem, the above expression becomes

$$
\left(\sum_{(h, k)} \xi_{h k}^{(T)} p^{h} q^{k}\right)^{\mu}\left(\sum_{(h, k)} \omega_{h k}^{(T)} p^{h} q^{k}\right)^{\nu}=\left[\pi^{(T)}(p)\right]^{\mu}\left[\rho^{(T)}(p)\right]^{\nu}=m(E) .
$$

The justification for this application of the multinomial theorem lies in the absolute convergence of the series for $\pi^{(T)}(p)$ and $\rho^{(T)}(p)$. To prove this, consider the case where $\mu=1$ and $\nu=0$. Under these conditions, the series for $\pi^{(T)}(p)$ would be obtained as the measure of $E$, without the necessity of applying the multinomial theorem. Since $m(E) \leqq 1$, the series converges absolutely. A similar argument applies to the series for $\rho^{(T)}(p)$.

It follows from this theorem that any finite transformation is admissible; for, in the case of a finite transformation, it follows from the condition of indeterminacy that the first digit of $v$ necessarily exists. Hence, for every rational $p$ in the interval $0<p<1, \pi^{(T)}(p)+\rho^{(T)}(p)=1$, since the left-hand member is the measure of all numbers $y$ in the unit interval. Since, for this case, $\pi^{(T)}(p)+\rho^{(T)}(p)$ is a polynomial in $p$, it must be identically 1 in $p$.

If $T$ belongs to $R$ but not to $R_{a}$, there will be certain numbers $y$ such that the corresponding number $v$ contains only a finite number of digits, or fails entirely to exist. If $T$ belongs to $R$, it follows from Theorem 2 that the measure of the set of numbers $y$ such that the corresponding number $v=T(u)$ has at least $m$ digits is*

$$
\sum_{s=0}^{m} C_{m, s}\left[\pi^{(T)}(p)\right]^{s}\left[\rho^{(T)}(p)\right]^{m-s}=\left[\pi^{(T)}(p)+\rho^{(T)}(p)\right]^{m} .
$$

Since the existence of the probability $p(v)$ requires that $v$ have an infinite number of digits, the measure of the set of numbers $y$ for which the existence of this probability is possible is

$$
\lim _{m \rightarrow \infty}\left[\pi^{(T)}(p)+\rho^{(T)}(p)\right]^{m},
$$

which has the value 1 or 0 , according as the expression within the brackets is equal to or less than unity. If $T$ is admissible, the value is, of course, 1 ; otherwise, it may be either 1 or 0 , as there exist transformations such that $\pi^{(T)}(p)+\rho^{(T)}(p)$ is unity for some values of $p$ and not for others. An example of such a transformation is given at the end of the paper.

3. Admissibility of numbers obtained through transformations of the set $R_{a}$. Although the probability functions $\pi^{(T)}(p)$ and $\rho^{(T)}(p)$ were shown in the preceding section to be the measures of the sets of numbers $y$ for which the

* $C_{m, 8}$ represents the number of combinations of $m$ things $s$ at a time. 
first digits of $v$ are 1 and 0 , respectively, the use of these functions as representing the probabilities of success and failure of the event associated with the number $v$ will not be justified until it has been shown that $p[T(u)]=\pi^{(T)}(p)$. To show this is the purpose of the next theorem.

THEOREM 3. For any transformation $T$ of $R_{a}$ and any rational $p,(0<p<1)$, the set of numbers $y$ corresponding to the set of all numbers $u$ such that $p[T(u)]$ $=\pi^{(T)}(p)$ has init measure. ${ }^{*}$

Let $V=\lim \sup _{\mu \rightarrow \infty}\left|p_{\mu}(v)-\pi^{(T)}(p)\right|$, where $v=T(u)$. Then, if $E(V>\epsilon)$ denotes the set of numbers $y$ such that $V>\epsilon$,

$$
E(V>0)=E(V>1 / 2)+E(V>1 / 3)+E(V>1 / 4)+\cdots .
$$

Hence the theorem will be proved if we can show that $m[E(V>\boldsymbol{\epsilon})]=0$ for every positive number $\epsilon$. We have

and hence

$$
E(V>\epsilon)=\lim _{\mu_{0} \rightarrow \infty} \sum_{\mu=\mu_{0}}^{\infty} E\left[\left|p_{\mu}(v)-\pi^{(T)}(p)\right|>\epsilon\right]
$$

$$
m[E(V>\epsilon)] \leqq \sum_{\mu=\mu_{0}}^{\infty} m\left\{E\left[\left|p_{\mu}(v)-\pi^{(T)}(p)\right|>\epsilon\right]\right\}
$$

for every positive integer $\mu_{0}$. The remainder of the proof consists in establishing the convergence of this series. From Theorem 2, it follows that

$$
m\left\{E\left[\left|p_{\mu}(v)-\pi^{(T)}(p)\right|>\epsilon\right]\right\}=\sum_{\left|s / \mu-\pi^{(T)}(p)\right|>\epsilon} C_{\mu, s}\left[\pi^{(T)}(p)\right]^{s}\left[1-\pi^{(T)}(p)\right]^{\mu-s}
$$

where the expression below the summation sign indicates that the summand is to be summed for all values of $s$ consistent with this inequality. Borel has proved $\dagger$ the convergence of all series of the form

$$
\sum_{\mu=1}^{\infty} \sum_{|s / \mu-p|>\epsilon} C_{\mu, s} p^{s} q^{\mu-s},
$$

where $p$ and $q$ are positive numbers and $p+q=1$. Hence the theorem follows.

THEOREM 4. For any transformation $T$ of $R_{a}$ and any rational $p,(0<p<1)$, the set of numbers $y$ corresponding to the set of all numbers $u$ such that $u$ is an element of $A(p) \ddagger$ and $T(u)$ is an element of $A\left[\pi^{(T)}(p)\right]$ has unit measure.

It is evident that $T_{\lambda}$ belongs to the set $R_{f}$-the set $P^{\left(T_{\lambda}\right)}$ consisting of all possible permutations of $n$ digits; and $\pi^{\left(T_{\lambda}\right)}(p)=p^{k}$. Moreover, it follows from

* This method was used by Copeland in similar theorems. See Copeland $[5,7]$.

$\dagger$ Borel [1].

$\ddagger A(p)$ is the set of all admissible numbers associated with the probability $p$. 
Theorem 1 that $T_{\lambda}[T(u)]=T_{\lambda}^{\prime}(u)$, where $T_{\lambda}^{\prime}$ belongs to $R_{a}$. By Theorem 3, the set of numbers $y$ such that $p\left[T_{\lambda}^{\prime}(u)\right] \neq\left[\pi^{(T)}(p)\right]^{k}$ has zero measure. Hence, the set for which the equality $p\left[T_{\lambda}^{\prime}(u)\right]=\left[\pi^{(T)}(p)\right]^{k}$ is not satisfied for every $\lambda$, that is, the set for which $T(u)$ does not belong to $A\left[\pi^{(T)}(p)\right]$ has zero measure. Since $R_{a}$ includes the identity transformation $T_{0}$, the set for which $u$ does not belong to $A(p)$ has zero measure.

This completes the justification of formulas (1) as the probabilities associated with numbers obtained from admissible numbers by transformations of the set $R_{a}$, where the original numbers are associated with rational probabilities. It is desirable and possible to extend these results to include the case of irrational probabilities. Such an extension will be the subject of the next section.

4. Admissibility of numbers associated with irrational probabilities. The extension of the properties of the set $R_{a}$ to include admissible numbers associated with all real probabilities is accomplished by the use of the property $G$, and by means of the following theorem.

THEOREM 5. Corresponding to every real number $\theta$ in the interval $0<\theta<1$, there exists a transformation $T$ of $R_{a}$ such that $\pi^{(T)}(1 / 2)=\theta$.

Let the number $\theta$ be represented as an infinite radix fraction in the scale of two.* Let the set $P^{(T)}$ consist of the permutations $c_{i},(i=1,2, \cdots)$, where $c_{i}$ consists of $(i-1)$ 1's followed by a single 0 . The subsets $P_{1}{ }^{(T)}$ and $P_{0}{ }^{(T)}$ are then formed as follows. If $\theta^{(i)}=1, c_{i}$ belongs to $P_{1}{ }^{(T)}$; otherwise, $c_{i}$ belongs to $P_{0}^{(T)}$. For every $h, \xi_{h 1}^{(T)}=\theta^{(h+1)}, \omega_{h 1}^{(T)}=1-\theta^{(h+1)}$, and $\xi_{h k}^{(T)}=\omega_{h k}^{(T)}=0$ for $k \neq 1$. Hence,

$$
\pi^{(T)}(1 / 2)=\sum_{i=1}^{\infty} \frac{\theta^{(i)}}{2^{i}}=\theta .
$$

Moreover, $\pi^{(T)}(p)+\rho^{(T)}(p)=\sum_{i=0}^{\infty} p^{i} q=1$, for $0<p<1$.

It can now be shown that transformations of the set $R_{a}$ give rise to admissible numbers even when applied to admissible numbers having irrational probabilities.

THEOREM 6. Corresponding to every transformation $T$ of $R_{a}$ and every $p$ in the interval $0<p<1$, there exists a nondenumerable subset $E$ of the set $A(p)$ such that, for every number $v$ of $E, T(v)$ belongs to $A\left[\pi^{(T)}(p)\right]$, and the corresponding set of numbers $T(v)$ is nondenumerable.

By Theorem 5, there exists a transformation $T^{\prime}$ of $R_{a}$ such that $\pi^{\left(T^{\prime \prime}\right)}(1 / 2)$ $=p$. Since $R_{a}$ has the property $G, T\left[T^{\prime}(u)\right]=T^{\prime \prime}(u)$, where $T^{\prime \prime}$ belongs to $R_{a}$.

${ }^{*}$ If $p$ is expressible as a finite sum of powers of two, so that two such representations are possible, it makes no difference which is employed. 
By Theorem 4, the sets of numbers $y$ in the scale of two corresponding to the set of numbers $u$ such that $T^{\prime}(u)$ does not belong to $A(p)$, and to the set of numbers $u$ such that $T^{\prime \prime}(u)$ does not belong to $A\left[\pi^{(T)}(p)\right]$, both have zero measure. Therefore, the set of numbers $y$ corresponding to the set of numbers $u$ such that $T^{\prime}(u)$ belongs to $A(p)$ and $T^{\prime \prime}(u)$ belongs to $A\left[\pi^{(T)}(p)\right]$ has unit measure. Let $E$ denote the corresponding set of numbers $v=T^{\prime}(u)$. By Theorem 2, the measure of the set of numbers $y$ corresponding to a given number $T^{\prime}(u)$ is

$$
\lim _{\mu \rightarrow \infty, \nu \rightarrow \infty} p^{\mu}(1-p)^{\nu}=0,
$$

and, similarly, the measure of the set of numbers $y$ corresponding to a given number $T^{\prime \prime}(u)$ is

$$
\lim _{\mu \rightarrow \infty, \nu \rightarrow \infty}\left[\Pi^{(T)}(p)\right]^{\mu}\left[\rho^{(T)}(p)\right]^{\nu}=0 .
$$

If either the set $E$ or the corresponding set of numbers $T(v)$ were denumerable, then the set of numbers $y$ such that $v$ belongs to $A(p)$ and $T(v)$ belongs to $A\left[\pi^{(T)}(p)\right]$ would have the measure zero, which has been proved false.

The formulas (1), obtained originally from the $a$ priori point of view, have now been justified in the light of the statistical definition of probabiiity, since they have been shown to be the actual limiting values of the success and failure ratios associated with numbers obtained from admissible numbers by transformations of the set $R_{a}$. Theorem 6 also furnishes a new proof for the existence of admissible numbers having all probabilities in the interval $0<p<1$.

5. Further properties of transformations of the set $R$. Certain additional properties of transformations of the sets $R$ and $R_{a}$ are contained in the following theorems.

THEOREM 7. For any transformation $T$ of $R$ and any $p$ in the interval $0<p<1, \pi^{(T)}(p)+\rho^{(T)}(p) \leqq 1$.

By Theorem 5 , there exists a transformation $T^{\prime}$ of $R_{a}$ such that $\pi^{\left(T^{\prime}\right)}(1 / 2)$ $=p$; and, since $R$ has the property $G, T\left[T^{\prime}(u)\right]=T^{\prime \prime}(u)$, where $T^{\prime \prime}$ belongs to $R$. Hence

$$
\pi^{(T)}(p)+\rho^{(T)}(p)=\pi^{\left(T^{\prime \prime}\right)}(1 / 2)+\rho^{\left(T^{\prime \prime}\right)}(1 / 2) .
$$

The right-hand member has at most the value 1 since it may be regarded as the measure of a set of numbers $y$ contained in the unit interval.

THEOREM 8. The sets $R$ and $R_{a}$ have the power of the continuum.

By Theorem 5, there corresponds to every number of the continuum a 
distinct transformation $T$ of $R_{a}$. It will now be shown that to every transformation of $R$ there corresponds a distinct number of the continuum. Assume that a one-to-one correspondence has been established between the set of all positive integers $\lambda$ and the set $P \equiv\left[c_{\lambda}\right]$ of all finite permutations of zeros and ones. Corresponding to any transformation $T$ of $R$, let the number $x_{T}$ be defined as follows: $x_{T}^{(2 \lambda-1)}=1$ if $c_{\lambda}$ belongs to $P_{1}^{(T)}$, and 0 otherwise; $x_{T}{ }^{(2 \lambda)}=1$ if $c_{\lambda}$ belongs to $P_{0}{ }^{(T)}$, and 0 otherwise.

6. Invariance of admissibility under transformations of the set $R_{a}$. By the definition of the admissible number, the property of admissibility is an absolute invariant under all transformations of the type $T_{\lambda}$. This is what is meant by the statement that the set $D_{a}$ of all such transformations is the fundamental set for the set $M_{a}$ of all admissible numbers. It was not, however, proved in $\$ 4$ that the result of applying a transformation of the set $R_{a}$ to any admissible number is always an admissible number. In fact, it is only reasonable to suppose that, if the set $D_{a}$ be increased by the addition of certain other transformations of $R_{a}$, the set $M_{a}$ will have to be decreased in order that the absolute invariance be preserved. It will be shown, however, that the set of numbers is not materially decreased by adding to the fundamental set any denumerable set of transformations of the set $R_{a}$. If $v$ belongs to $A(p)$ and $T(v)$ belongs to $A\left[\pi^{(T)}(p)\right]$, the admissibility of $v$ is said to be regularly invariant under the transformation $T$.

Theorem 9. If $D$ is any denumerable subset of $R_{a}$, there exists a set $M$, consisting of admissible numbers and having the power of the continuum, such that, for every number of $M$, the property of admissibility is regularly invariant under every transformation of $D$.

Let the set $D$ consist of the transformations $T_{1}, T_{2}, T_{3}, \cdots$, and let $T_{0}$ denote the identity transformation. For every $p$ in the interval $0<p<1$, there exists, by Theorem 5, a transformation $T$ of $R_{a}$ such that $\pi^{(T)}(1 / 2)=p$. Let $N_{p}^{(i)}$ denote the set of numbers $y$ in the scale of two corresponding to the set of all numbers $u$ such that $T_{i}(v)=T_{i}[T(u)]$ does not belong to $A\left[\pi^{\left(T_{i}\right)}(p)\right]$. Then, by Theorem $4, m\left(N_{p}^{(i)}\right)=0$. Hence, $m C\left[\sum_{i=0}^{\infty} N_{p}^{(i)}\right]=1$. Therefore, the set $M_{p}$ of all numbers $v=T(u)$ associated with the numbers $y$ of the set $C\left[\sum_{i=0}^{\infty} N_{p}^{(i)}\right]$ is, by the reasoning of Theorem 6, nondenumerable, and therefore nonvacuous. Let the set $M$ consist of all the sets $M_{p}$ for all probabilities $p$ in the unit interval. It follows at once from the method of construction of $M$ that the admissibility of every number of $M$ is regularly invariant under every transformation of $D . M$ has the power of the continuum since each $M_{p}$ contains at least one number, which cannot belong to any other $M_{p}$.

The characteristic property of the numbers of $M$ is not mere admissibil- 
ity, but regular invariance of admissibility under the transformations of $D$. If thorough consistency is desired, not merely invariance of admissibility, but invariance of this characteristic property, should be demanded of the numbers of $M$. In other words, the result of applying a transformation of $D$ to a number of $M$ should be not merely an admissible number with the appropriate probability, but a number of $M$. In general, this type of invariance can be secured only if $D$ has the property $G$. However, it is always possible to increase the set $D$ so that it will have this property, without sacrificing denumerability. To accomplish this, add to $D$ every transformation which is the resultant of any finite number of transformations of $D$. Examples of denumerable subsets of $R_{a}$ having the property $G$ are the sets $R, R_{f}$, and $D_{a}$. For all such sets the following theorem holds.

Theorem 10. If $D$ is any denumerable subset of $R_{a}$ having the property $G$, there exists a set $M$, consisting of admissible numbers and having the power of the continuum, such that the result of applying successively to a number of $M$ any finite number of transformations of $D$ is a number of $M$, the property of admissibility being regularly invariant under all such transformations.

Let $\dot{M}$ be defined as in Theorem 9; and suppose there is a number $u$ of $M$ such that, by a finite number of transformations of $D$, it is possible to obtain from $u$ a number $v$ which does not belong to $M$. Since $D$ has the property $G$, there is a transformation $T$ of $D$, such that $v=T(u)$. Since $v$ does not belong to $M$, there is a transformation $T^{\prime}$ of $D$ such that $T^{\prime}(v)$ does not belong to $A\left\{\pi^{\left(T^{\prime}\right)}[p(v)]\right\}$. But

$$
T^{\prime}(v)=T^{\prime}[T(u)]=T^{\prime \prime}(u),
$$

where $T^{\prime \prime}$ belongs to $D$. This contradicts Theorem 9. The regular invariance of admissibility is an immediate consequence of the method of construction of $M$.

Any denumerable subset $D$ of $R_{a}$ may, therefore (with additions if necessary), play the same role in the definition of a system of numbers as the set $D_{a}$ in the definition of admissible numbers. The characteristic property of such a system would be invariance of admissibility under the transformations of $D$. This characteristic property is itself invariant under the transformations of $D$, so that the system of numbers so defined is, in a sense, closed with respect to the transformations of $D$. It is a question whether there exists a number for which admissibility is regularly invariant under all the transformations of $R_{a}$.

7. Illustrations. (i) Let a series of games be so arranged that $A$ wins the series if he wins a total of $r$ games before $B$ wins $s$ games; otherwise $B$ wins the series. Let $u$ represent the sequence of games, the digit 1 denoting a game 
won by $A$ and 0 by $B$, let $v$ represent the resulting sequence of rubbers or series of games, and let $T$ denote the transformation such that $v=T(u)$. In this case, $\xi_{r k}^{(T)}=C_{r+k-1, k}$ for $k<s$, and 0 for $k \geqq s$; and $\xi_{h k}^{(T)}=0$ for $h \neq r$. Similarly, $\omega_{h s}^{(T)}=C_{h+s-1, h}$ for $h<r$ and 0 for $h \geqq r ;$ and $\omega_{h k}^{(T)}=0$ for $k \neq s$. Hence,

$$
\pi^{(T)}(p)=\sum_{k=0}^{s-1} C_{r+k-1, k} p^{r} q^{k}, \quad \rho^{(T)}(p)=\sum_{h=0}^{r-1} C_{h+s-1, h} p^{h} q^{s} .
$$

Since this transformation satisfies the condition of indeterminacy, it belongs to $R_{f}$, and therefore to $R_{a}$. An illustration of this situation is found in the game of bridge, where $u$ represents a sequence of "games" and $v$ the resulting sequence of rubbers. Since the rubber is won by the side first winning two games, $r=s=2$, and

$$
\pi^{(T)}(p)=p^{2}+2 p^{2} q, \quad \rho^{(T)}(p)=q^{2}+2 p q^{2} .
$$

(ii) Let a series of games be so arranged that $A$ wins the series if and when the number of games won by him exceeds by $m$ the number won by $B$, provided the number of games won by $B$ has not previously exceeded by $n$ the number won by $A$. Similarly, $B$ wins if he secures a lead of $n$ games before $A$ is $m$ games ahead. The series is assumed to be continued until one of the players wins. Evidently this is not a finite transformation, since there is no upper limit to the number of games which may be necessary. It does, however, satisfy the condition of indeterminacy, and it can be shown* that

$$
\pi^{(T)}(p)=\frac{p^{m}\left(p^{n}-q^{n}\right)}{p^{m+n}-q^{m+n}}, \quad \rho^{(T)}(p)=\frac{q^{n}\left(p^{m}-q^{m}\right)}{p^{m+n}-q^{m+n}}
$$

if $p \neq q$; and if $p=q=1 / 2$,

$$
\pi^{(T)}(1 / 2)=\frac{n}{m+n}, \quad \rho^{(T)}(1 / 2)=\frac{m}{m+n} .
$$

If $m=n$, both expressions reduce to the simpler form

$$
\pi^{(T)}(p)=\frac{p^{m}}{p^{m}+q^{m}}, \quad \rho^{(T)}(p)=\frac{q^{m}}{p^{m}+q^{m}} .
$$

In all cases, $\pi^{(T)}(p)+\rho^{(T)}(p) \equiv{ }_{p} 1$, so that $T$ belongs to $R_{a}$. This situation is illustrated by the case of two players matching pennies, one player starting with $m$ and the other with $n$ pennies, the game terminating when either player has lost all his pennies.

(iii) The following is an example of a transformation which belongs to the

* Uspensky [1, pp. 139-142]. 
set $R$ but is not admissible. It also illustrates the fact that $\pi^{(T)}(p)+\rho^{(T)}(p)$ may be unity for some values of $p$ and not for others. Let the set of specified permutations consist of all those in which the number of ones exceeds the number of zeros (after superfluous permutations have been eliminated). Each such permutation will be a " 0 permutation" or " 1 permutation" according as the number of 0 's preceding the first 1 in the permutation is even or odd. It can be shown* that $\pi^{(T)}(p)+\rho^{(T)}(p)$ is $p / q$ for $p \leqq 1 / 2$, and 1 for $p \geqq 1 / 2$.

\section{REFERENCES}

E. BOREL

1. Traité du Calcul des Probabilités et de ses Applications, vol. 2, no. 1, chap. 1, Paris, 1926.

A. H. Copeland

1. Admissible numbers in the theory of probability, American Journal of Mathematics, vol. 50 (1928), pp. 535-552.

2. Independent event histories, American Journal of Mathematics, vol. 51 (1929), pp. 612-618.

3. The theory of probability from the point of view of admissible numbers, Annals of Mathematical Statistics, vol. 3 (1932), pp. 143-156.

4. A matrix theory of measurement, Mathematische Zeitschrift, vol. 37 (1933), pp. 542-555.

5. Point set theory applied to the random selection of the digits of an admissible number, American Journal of Mathematics, vol. 58 (1936), pp. 181-192.

6. Probabilities and predictions, Erkenntnis, vol. 6 (1937), pp. 189-203.

7. Consistency of the conditions determining Kollektivs, these Transactions, vol. 42 (1937), pp. 333-357.

K. DöRGE

1. Zu der R. v. Mises gegebenen Begründung der Wahrscheinlichkeitsrechung, Mathematische Zeitschrift, vol. 32 (1930), pp. 232-258.

E. KAMKE

1. Über neuere Begründungen der Wahrscheinlichkeitsrechnung, Jahresbericht der deutschen Mathematiker-Vereinigung, vol. 42 (1932), pp. 14-27.

2. Einführung in der Wahrscheinlichkeitstheorie, Leipzig, 1932.

R. voN MISES

1. Grundlagen der Wahrscheinlichkeitsrechnung, Mathematische Zeitschrift, vol. 5 (1919), pp. 52-99.

2. Vorlesungen aus dem Gebiete der angewandte Mathematik, vol. 1, Wahrscheinlichkeit, Leipzig, 1931.

3. Über Zahlenfolgen die ein kollektiv-ähnliches Verhalten zeigen, Mathematische Annalen, vol. 108 (1933), pp. 757-772.

4. Wahrscheinlichkeit, Statistik, und Wahrheit, Vienna, 1936.

H. REICHENBACH

1. Axiomatik der Wahrscheinlichkeitsrechnung, Mathematische Zeitschrift, vol. 34 (1932), pp. 568-619.

2. Wahrscheinlichkeitslehre, Leiden, 1935.

3. Les fondements logiques du calcul des probabilités, Annales de l'Institut Henri Poincaré, vol. 7 (1937), pp. 568-619.

E. TORNIER

1. Wahrscheinlichkeitsrechnung und Zahlentheorie, Journal für die reine und angewandte Mathematik, vol. 160 (1929), pp. 177-198.

*Uspensky [1, p. 142]. 
2. Die Axiome der Wahrscheinlichkeitsrechnung, Journal für die reine und angewandte Mathematik, vol. 163 (1930), pp. 45-64.

J. V. USPENSKY

1. Irtroduction to Mathematical Probability, chap. 8, New York, 1937.

C. DE la Vallée Poussin

1. Sur l'intégrale de Lebesgue, these Transactions, vol. 16 (1915), pp. 435-501.

2. Intégrales de Lebesgue, Paris, 1916.

A. WALD

1. Die Widerspruchsfreiheit des Kollektivbegriffes der Wahrscheinlichkeitsrechnung, Ergebnisse eines mathematischen Kolloquiums, vol. 8 (1935-1936), pp. 38-72.

UNIVERSITY OF MICHIGAN, ANN ARBor, Mich. 